\title{
Intellectual disability-facial dysmorphism syndrome due to SETD5 haploinsufficiency
}

INSERM

\section{Source}

INSERM. (1999). Orphanet: an online rare disease and orphan drug data base. Intellectual disability-facial dysmorphism syndrome due to SETD 5 haploinsufficiency. ORPHA:404440

Intellectual disability-facial dysmorphism syndrome due to SETD5 haploinsufficiency is a rare, syndromic intellectual disability characterized by intellectual disability of various severity, hypotonia, feeding difficulties, dysmorphic features, autism and behavioral issues. Growth retardation, congenital heart anomalies, gastrointestinal and genitourinary defects have been rarely associated. 